\title{
mTOR and tau phosphorylated proteins in the hippocampal tissue of rats with type 2 diabetes and Alzheimer's disease
}

\author{
YUN-QING MA, DONG-KE WU and JIAN-KUN LIU \\ Department of Endocrinology, The First Affiliated Hospital of Nanchang University, \\ Nanchang 330006, P.R. China
}

Received July 4, 2012; Accepted November 1, 2012

DOI: $10.3892 / \mathrm{mmr} .2012 .1186$

\begin{abstract}
This study explored the effects of mammalian target of rapamycin (mTOR) on the increased risk of developing Alzheimer's disease (AD) in rats with type 2 diabetes mellitus (T2DM). Male Sprague-Dawley rats were randomly divided into four groups: control, T2DM, AD and T2DM+AD. Changes in the learning and memory abilities of the rats were observed using the Morris water maze. mTOR activity and tau protein hyperphosphorylation in the hippocampus were analyzed by immunohistochemical staining and RT-PCR. The learning and memory abilities of the experimental rats were weakened compared with those of the control group. The T2DM+AD group revealed significant changes over the T2DM and AD groups. Compared with the control, T2DM and AD groups, the mTOR protein and mRNA levels, hyperphosphorylation of tau protein and total tau protein mRNA levels were significantly increased in the T2DM+AD group. T2DM may excessively activate mTOR in the hippocampal tissue by impairing insulin signaling, thereby increasing the extent of tau hyperphosphorylation and promoting the occurrence of $\mathrm{AD}$.
\end{abstract}

\section{Introduction}

Type 2 diabetes mellitus (T2DM) and Alzheimer's disease (AD) are common chronic diseases in the elderly. The prevalence of T2DM and AD is increasing sharply due to socio-demographic aging and lifestyle changes $(1,2)$. Epidemiologic evidence suggests an association between T2DM and AD. Other studies $(3,4)$ have shown that patients with T2DM are at greater risk of developing AD than non-diabetic patients. Thus, AD is occasionally referred to as type 3 diabetes mellitus (5). Although the specific mechanism

Correspondence to: Professor Yun-Qing Ma, Cadre Ward, Department of Endocrinology, The First Affiliated Hospital of Nanchang University, No. 17 Yongwai Main Street, Nanchang 330006, P.R. China

E-mail: qingyun0706@sina.com

Key words: mTOR, hyperphosphorylation of tau protein, type 2 diabetes mellitus, Alzheimer's disease, insulin resistance by which T2DM increases the risk of AD is unclear, it has been proposed that insulin resistance (IR) is significant in the pathogenesis of AD. Studies have affirmed that IR is an independent risk factor for $\mathrm{AD}(6,7)$. The mammalian target of rapamycin (mTOR) is a key regulatory protein of the phosphoinositide 3-OH kinase/protein kinase $\mathrm{B}$ insulin signal transduction pathway (PI3K/PKB pathway). mTOR is able to adjust insulin sensitivity by providing feedback and maintaining the $\beta$-cell function of the islets of Langerhans. It is essential in the insulin signaling process (8). Certain studies (9-11) have indicated that mTOR is significantly involved in the development and progression of IR and T2DM. Other studies have shown that the hyperphosphorylation of tau, which is neurotoxic, increases with the activation of $\operatorname{mTOR}(12,13)$. However, the exact reason for this increase remains unclear. Impairment of insulin signaling induces IR in the brain, which promotes the onset of AD. Whether T2DM results in the excessive activation of mTOR, and thus insulin signaling damage, is unclear. No related studies have been reported to date. In the current study, behavioral changes, mTOR activity and tau protein phosphorylation in the hippocampal tissue were observed following the establishment of T2DM, AD and T2DM+AD models in rats. The models established in this study have suggested a role for mTOR in the increased risk of developing AD in T2DM rats.

AD is called central diabetes mellitus by some scholars (7). However, the reason for this finding is not yet clear. mTOR is involved in the development and progression of IR and T2DM. The characteristic pathological changes leading to the occurrence and development of $\mathrm{AD}$ are triggered by the excessive phosphorylation of tau protein. Thus, the excessive phosphorylation of tau is the key factor which causes the disease (13). The phosphorylation of the tau protein is regulated by protein kinases and protein phosphatases (including cyclin-dependent kinase 5 and glycogen synthase kinase 3) (12). Earlier studies $(14,15)$ have demonstrated that mTOR may be one of the kinases associated with excessive tau protein phosphorylation.

mTOR may be the common kinase involved in insulin signal transfer and protein phosphorylation. However, no studies have explored whether the impairment of insulin signaling results in excessive mTOR activity to induce excessive tau protein phosphorylation and, consequently, AD. This study used a T2DM+AD rat model to investigate the expression of mTOR in AD and its role in the increased risk of developing $\mathrm{AD}$ in T2DM rats. 


\section{Materials and methods}

Animals and grouping. Healthy male rats, which were clean grade and weighing $200 \pm 20 \mathrm{~g}$, were provided by the Jiangxi University of Traditional Chinese Medicine. This study was carried out in strict accordance with the recommendations of the Guide for the Care and Use of Laboratory Animals of the National Institutes of Health. The animal use protocol was reviewed and approved by the Institutional Animal Care and Use Committee of the Jiangxi University of Traditional Chinese Medicine. A Certificate of Conformity: SCXK (Jiangxi) 2005-0001 was obtained for the experimental animals. Rats that reacted sensitively or too slowly and had poor swimming posture or congenital dementia were eliminated using the Morris water maze. After one week of adaptive feeding, 50 qualified rats were divided into two groups: the normal control $(n=23)$ and T2DM $(n=27)$ groups. The rat model of T2DM was established via a single intraperitoneal injection of streptozotocin (STZ; Sigma, St. Louis, MO, USA) and the provision of a high-fat and high-carbohydrate diet. When the T2DM rat model was successfully established, the normal control group was divided into two groups: control $(\mathrm{n}=11)$ and $\mathrm{AD}(\mathrm{n}=12)$. The T2DM group was divided into the T2DM ( $n=12)$ and T2DM+AD $(n=15)$ groups. The AD rat model was established by injecting $\mathrm{A} \beta 1-40$ into the bilateral hippocampus at stereotaxic coordinates. All rats ate and drank freely during the experimental period.

T2DM rat model. After one week of adaptive feeding, the T2DM group was provided with a high-fat and high-carbohydrate diet for eight weeks, and then injected with a single intraperitoneal STZ dose of $30 \mathrm{mg} / \mathrm{kg}$. In comparison, the control group received a single intraperitoneal injection of citric acid buffer at the same volume. After one week, blood glucose levels were measured via the tail vein. A fasting plasma glucose level $\geq 16.7 \mathrm{mmol} / 1$ was considered to indicate the successful establishment of a type 2 diabetes model.

$A D$ rat model. The rats were anesthetized via an intraperitoneal injection of chloral hydrate $(10 \% 3 \mathrm{ml} / \mathrm{kg})$. Their heads were fixed with a stereotaxic locator. Skin preparation and sterilization were performed. A $2-\mathrm{cm}$ incision along the midline of the skull top was made, and the skull was subsequently exposed by separating the periosteum. The anterior fontanelle, which was the basis point, was identified in the parietal lobe. A needle point was located $3.0 \mathrm{~mm}$ behind the anterior fontanelle and $2.0 \mathrm{~mm}$ next to the midline based on the stereotactic atlas of the rat brain. The skull was opened using a miniature dental drill. A needle was vertically inserted $2.9 \mathrm{~mm}$ into the hippocampal tissue. Condensed matter $(1 \mu \mathrm{l})$ of A $1-40$, which was placed into a $5 \mu 1$ micro injector, was injected into the two sides of the hippocampal tissue. The needle was slowly withdrawn after remaining in the brain for $10 \mathrm{~min}$. The skin covering the area was subsequently sutured. The AD model was thereby established. Using the same method, an equal volume of sterile saline was injected into the two sides of the hippocampal tissue of the control and T2DM rats. The Morris water maze test was performed one week after injection. The standard was the upper limit of $95 \%$ of the control rats. The rats that exceeded this limit were the demented rats. Thus, the AD rat model was successfully established.

$T 2 D M+A D$ rat model. Four weeks after the successful establishment of the T2DM rat model, a composite model of AD was generated following the method previously described.

Morris water maze experiment. The Morris water maze test was conducted for seven days, beginning one week after injection of the hippocampus. The directional navigation test was performed in the first six days of the experiment. The performance of each rat from swimming in the water to climbing the platform within $60 \mathrm{sec}$ was recorded for the latency period. If the rat found the platform within $60 \mathrm{sec}$, then it was allowed to stand on the platform for $10 \mathrm{sec}$; if not, it was aided in arriving at the platform with a test-stick, after which, it was also allowed to stand on the platform for $10 \mathrm{sec}$. The time taken to reach the platform was recorded as its latency period. Each rat was trained four times every day, twice in the morning and twice in the afternoon. The seventh day of the test was allotted for the space exploration test. The platform was removed and the rat was placed into the second quadrant of the water maze. The number of times the rat crossed to the original location of the platform within $120 \mathrm{sec}$ was then recorded.

Biochemical indicator testing. Blood serum samples were drawn from the inferior vena cava. The samples were sent to the Inspection Center at the First Affiliated Hospital of Nanchang University to determine the fasting blood glucose (FBG), total cholesterol (TC) and triglyceride (TG) levels.

Immunohistochemistry. The rats were sacrificed by decapitation. The bilateral hippocampal organization was stripped and fixed with $4 \%$ neutral formaldehyde. The immunohistochemical method (sp) was used to detect mTOR and phosphorylated tau protein (Ser202). Three tissue slices of the hippocampal organization of each rat were observed under a microscope at $\mathrm{x} 400$. Four non-overlapping fields were then randomly selected. The number of positive cells in each view was counted and, using the Image-Pro Plus v 5.5 image analysis system, the percentage of positive-reaction cells from the total number of cells in each field of vision was calculated and averaged.

$R T$-PCR. After removal, the bilateral hippocampal organizations were immediately preserved in liquid nitrogen, and then placed in an ice box at $-80^{\circ} \mathrm{C}$. RT-PCR was used to detect the mTOR mRNA content and total tau protein mRNA expression. The mTOR primers were: upstream, 5'-ATG ACG AGA CCC AGG CTA AG-3'; and downstream, 5'-GCC AGT CCT CTA CAA TAC GC-3'. The amplification segment was $\sim 387 \mathrm{bp}$. The tau protein primers were: upstream 5'-CGG CGT AAG CAA AGA CA-3'; and downstream, 5'-TGT AGC CGC TTC GTT CT-3'. The amplification segment was $\sim 215 \mathrm{bp}$. The GAPDH primers were: upstream, 5'-CCT CTG GAA AGC TGT GGC GT-3'; and downstream, 5'-TTG GAG GCC ATG TAG GCC AT-3'. The amplification segment was $\sim 430 \mathrm{bp}$. An integrated gel imaging and analysis system was used for the spectrophotometric scanning and analysis of the bands. The gene and reference gene absorbance values indicate the relative strengths of the genetic expression. 
Table I. Biochemical indicators (mean $\pm \mathrm{s}$ ).

\begin{tabular}{lccll}
\hline Group & Cases & $\begin{array}{c}\text { FBG } \\
(\mathrm{mmol} / \mathrm{l})\end{array}$ & \multicolumn{1}{c}{$\begin{array}{c}\text { TG } \\
(\mathrm{mmol} / \mathrm{l})\end{array}$} & $\begin{array}{c}\text { TC } \\
(\mathrm{mmol} / \mathrm{l})\end{array}$ \\
\hline Control & 11 & $4.85 \pm 0.49$ & $0.75 \pm 0.06$ & $1.06 \pm 0.13$ \\
T2DM & 10 & $22.57 \pm 2.90^{\mathrm{a}}$ & $1.47 \pm 0.10^{\mathrm{a}}$ & $2.47 \pm 0.06^{\mathrm{a}}$ \\
AD & 9 & $5.06 \pm 0.66^{\mathrm{b}}$ & $0.71 \pm 0.08^{\mathrm{b}}$ & $1.01 \pm 0.08^{\mathrm{b}}$ \\
T2DM+AD & 9 & $24.53 \pm 2.21^{\text {acd }}$ & $1.57 \pm 0.12^{\text {acd }}$ & $2.57 \pm 0.13^{\text {acd }}$ \\
\hline
\end{tabular}

T2DM, type 2 diabetes mellitus; AD, Alzheimer's disease; FBG fasting blood glucose; TG, triglyceride; TC, total cholesterol. ${ }^{a} \mathrm{P}<0.01$ compared with the control group; ${ }^{b} \mathrm{P}<0.01,{ }^{\mathrm{c}} \mathrm{P}<0.05$ compared with the T2DM group; ${ }^{\mathrm{d}} \mathrm{P}<0.01$ compared with the $\mathrm{AD}$ group.

Table II. Times of crossing to the former platform location in $120 \sec ($ mean $\pm \mathrm{s})$.

\begin{tabular}{lcc}
\hline Group & Cases & No. of crossings \\
\hline Control & 11 & $11.91 \pm 1.81$ \\
T2DM & 10 & $7.10 \pm 0.99^{\mathrm{a}}$ \\
AD & 9 & $3.00 \pm 0.71^{\mathrm{ab}}$ \\
T2DM+AD & 9 & $1.78 \pm 0.83^{\mathrm{a}-\mathrm{c}}$ \\
\hline
\end{tabular}

T2DM, type 2 diabetes mellitus; AD, Alzheimer's disease. ${ }^{a} \mathrm{P}<0.01$ compared with the control group; ${ }^{b} \mathrm{P}<0.01$ compared with the $\mathrm{T} 2 \mathrm{DM}$ group; ${ }^{\mathrm{c}} \mathrm{P}<0.05$ compared with the $\mathrm{AD}$ group.

Statistical methods. SPSS 13.0 statistical software was used to analyze the results. Data are expressed as mean \pm standard deviation. Single factor analysis of variance was used to compare the differences among and between the groups. When the differences were significant, SNK-q inspection was used to compare the two groups. $\mathrm{P}<0.05$ was considered to indicate a statistically significant result.

\section{Results}

Survival rate. At the end of the experiment, 39 rats survived. The numbers of surviving rats in the normal control, T2DM, $\mathrm{AD}$ and T2DM+AD groups were 11, 10, 9 and 9, respectively. The successful modeling rates were $100,83,75$ and $60 \%$, respectively.

Biochemical indicators. Compared with the control and AD groups, the FBG, TC and TG levels of the T2DM and T2DM+AD groups were elevated $(\mathrm{P}<0.01)$. No significant difference was observed between the control and AD groups. However, the FBG, TC and TG levels in the T2DM+AD group were higher than those in the T2DM group $(\mathrm{P}<0.05$; Table I).

Behavioral changes. In the Morris water maze place navigation test, the average escape latency of the rats in each group was gradually shortened. The latency of the control group was the most evidently reduced. Compared with the control group, the latencies of the T2DM, AD and T2DM+AD groups were
Table III. Immunohistochemistry results for mTOR (mean $\pm s$ ).

\begin{tabular}{lcl}
\hline Group & Cases & mTOR \\
\hline Control & 11 & $0.27 \pm 0.04$ \\
T2DM & 10 & $0.49 \pm 0.12^{\mathrm{a}}$ \\
$\mathrm{AD}$ & 9 & $0.21 \pm 0.05^{\mathrm{b}}$ \\
T2DM+AD & 9 & $0.62 \pm 0.07^{\mathrm{acd}}$ \\
\hline
\end{tabular}

mTOR, mammalian target of rapamycin; T2DM, type 2 diabetes mellitus; AD, Alzheimer's disease. ${ }^{a} \mathrm{P}<0.05$ compared with the control group; ${ }^{b} \mathrm{P}<0.05,{ }^{\mathrm{c}} \mathrm{P}<0.01$ compared with the $\mathrm{T} 2 \mathrm{DM}$ group; ${ }^{\mathrm{d}} \mathrm{P}<0.01$ compared with the $\mathrm{AD}$ group.

Table IV. Immunohistochemistry results for the hyperphosphorylation of tau (Ser202) (mean $\pm s$ ).

\begin{tabular}{lcc}
\hline Group & Cases & Tau \\
\hline Control & 11 & $0.14 \pm 0.05$ \\
T2DM & 10 & $0.22 \pm 0.14$ \\
AD & 9 & $0.57 \pm 0.06^{\mathrm{ab}}$ \\
T2DM+AD & 9 & $0.73 \pm 0.08^{\mathrm{ac}}$ \\
\hline
\end{tabular}

T2DM, type 2 diabetes mellitus; AD, Alzheimer's disease. ${ }^{\mathrm{a}} \mathrm{P}<0.01$ compared with the control group; ${ }^{\text {}} \mathrm{P}<0.01$ compared with the $\mathrm{T} 2 \mathrm{DM}$ group; ${ }^{\mathrm{c}} \mathrm{P}<0.01$ compared with the $\mathrm{AD}$ group.

not significantly shortened. The latency trends among these groups were similar. In the space exploration experiments, the control rats swam in the quadrant containing the platform. However, all rats in the experimental groups swam in all platform quadrants. Compared with those in the control and T2DM groups, the number of times the AD and T2DM+AD rats crossed to the initial platform position decreased significantly $(\mathrm{P}<0.01)$. Significant differences were observed between the control and T2DM groups $(\mathrm{P}<0.01)$ as well as between the $\mathrm{AD}$ and $\mathrm{T} 2 \mathrm{DM}+\mathrm{AD}$ groups $(\mathrm{P}<0.05$; Table II $)$.

Immunohistochemistry results. mTOR-positive cells, which exhibit a brown cytoplasmic color, were observed near the injection site on the hippocampal nerve cells of each rat. Compared with the control and AD groups, the mTOR levels in the hippocampal tissues of the T2DM and T2DM+AD groups were significantly increased $(\mathrm{P}<0.05)$. However, no significant difference was observed between the control and AD groups. A significant difference was observed between the T2DM+AD and T2DM groups ( $\mathrm{P}<0.01$; Table III and Fig. 1).

The tau (Ser202) hyperphosphorylation-positive cells also underwent brown cytoplasmic staining. These cells were also observed near the injection site on the hippocampal nerve cells of each rat. Compared with the control and T2DM groups, the tau hyperphosphorylation levels in the hippocampal tissues of the AD and T2DM+AD groups were significantly increased $(\mathrm{P}<0.01)$. No significant difference was observed between the control and T2DM groups or between the T2DM+AD and AD groups ( $\mathrm{P}<0.01$; Table IV and Fig. 2). 
Table V. Gene expression of mTOR (mean $\pm s)$.

\begin{tabular}{lcc}
\hline Group & Cases & mTOR \\
\hline Control & 11 & $0.62 \pm 0.07$ \\
T2DM & 10 & $0.71 \pm 0.12^{\mathrm{a}}$ \\
AD & 9 & $0.61 \pm 0.01^{\mathrm{b}}$ \\
T2DM+AD & 9 & $0.88 \pm 0.05^{\text {acd }}$ \\
\hline
\end{tabular}

mTOR, mammalian target of rapamycin; T2DM, type 2 diabetes mellitus; AD, Alzheimer's disease. ${ }^{a} \mathrm{P}<0.05$ compared with the control group; ${ }^{b} \mathrm{P}<0.05,{ }^{\mathrm{c}} \mathrm{P}<0.01$ compared with the $\mathrm{T} 2 \mathrm{DM}$ group; ${ }^{\mathrm{d}} \mathrm{P}<0.01$ compared with the $\mathrm{AD}$ group.
Table VI. Gene expression of total tau protein (mean $\pm \mathrm{s})$.

\begin{tabular}{lrc}
\hline Group & Cases & Tau \\
\hline Control & 11 & $0.18 \pm 0.02$ \\
T2DM & 10 & $0.22 \pm 0.06$ \\
AD & 9 & $0.36 \pm 0.09^{\mathrm{ab}}$ \\
T2DM+AD & 9 & $0.54 \pm 0.05^{\mathrm{a}-\mathrm{c}}$ \\
\hline
\end{tabular}

T2DM, type 2 diabetes mellitus; $\mathrm{AD}$, Alzheimer's disease. ${ }^{\mathrm{a}} \mathrm{P}<0.01$ compared with the control group; ${ }^{\mathrm{P}}<0.01$ compared with the T2DM group; ${ }^{\mathrm{c}} \mathrm{P}<0.01$ compared with the $\mathrm{AD}$ group.

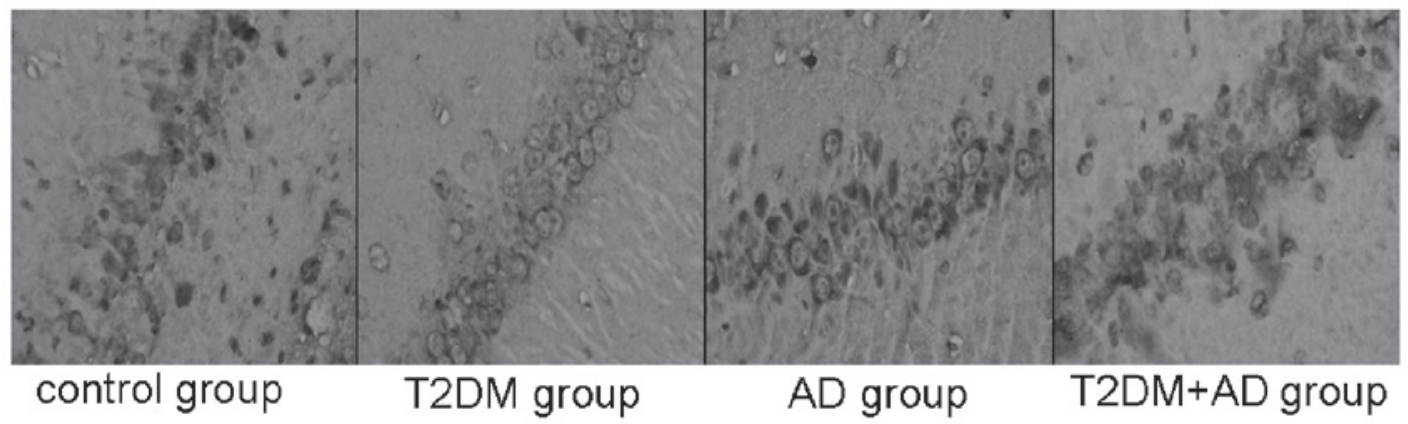

Figure 1. Immunohistochemistry results for mammalian target of rapamycin (mTOR; x400). T2DM, type 2 diabetes mellitus; AD, Alzheimer's disease.

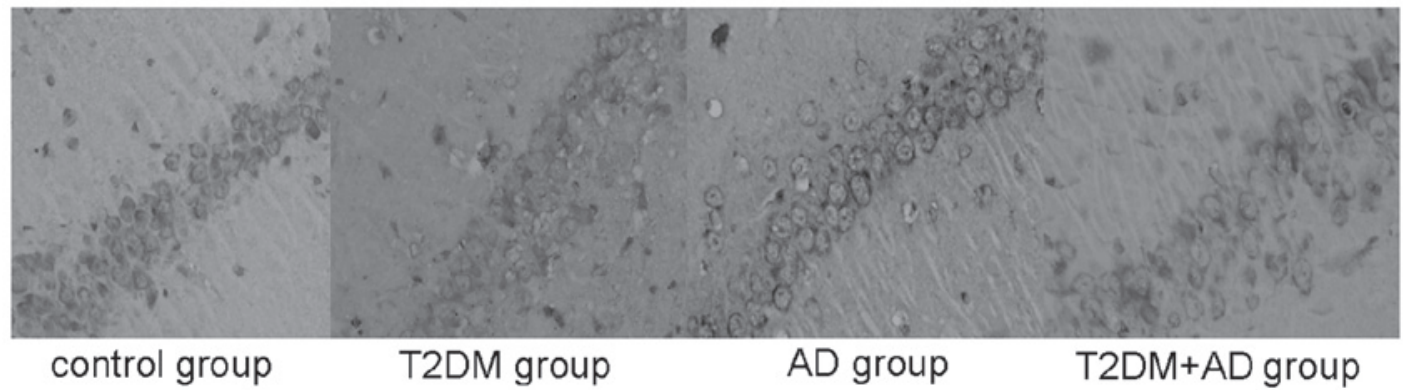

Figure 2. Immunohistochemistry results of hyperphosphorylation of tau (Ser202) (x400). T2DM, type 2 diabetes mellitus; AD, Alzheimer's disease.

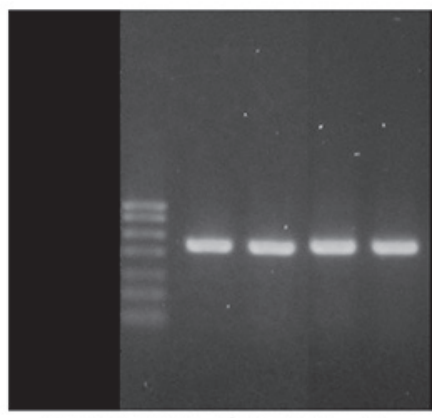

GAPDH (430 bp)

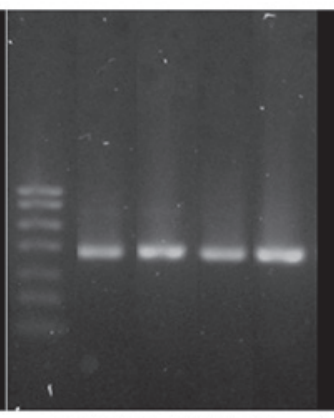

$\operatorname{mTOR}(387 \mathrm{bp})$

Figure 3. Gene expression of mammalian target of rapamycin (mTOR RT-PCR). Bands from left to right: marker, control group, type 2 diabetes mellitus (T2DM) group, Alzheimer's disease (AD) group and T2DM+AD group. Marker, from top to bottom: 700, 600, 500, 400, 300, 200 and $100 \mathrm{bp}$.

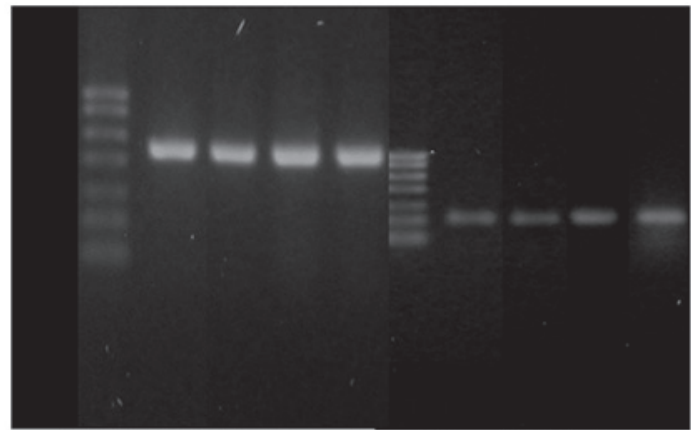

\section{GAPDH (430 bp) Tau (215 bp)}

Figure 4. Gene expression of total tau protein (RT-PCR). The bands in each figure from left to right are: marker, control group, type 2 diabetes mellitus (T2DM) group, Alzheimer's disease (AD) group and T2DM+AD group. Marker, from top to bottom: 700, 600, 500, 400, 300, 200 and $100 \mathrm{bp}$. 
mTOR and tau protein gene expression. The mTOR mRNA levels in the hippocampal tissues of the T2DM and T2DM+AD groups were significantly increased compared with those in the control and AD groups $(\mathrm{P}<0.05)$. No significant difference was observed between the control and AD groups. However, a significant difference was observed between the T2DM+AD and T2DM groups $(\mathrm{P}<0.01$; Table V and Fig. 3).

The total tau protein mRNA levels in the hippocampal tissues of the AD and T2DM+AD groups were significantly increased compared with those in the control and T2DM groups $(\mathrm{P}<0.01)$. No significant difference was observed between the control and T2DM groups. However, a significant difference was observed between the T2DM+AD and AD groups ( $\mathrm{P}<0.01$; Table VI and Fig. 4).

\section{Discussion}

The Morris water maze test revealed that the T2DM rats crossed to the platform significantly fewer times than the control rats. Thus, T2DM may degenerate rat memory and cognition abilities. Furthermore, compared with the AD rats, the number of times the T2DM+AD rats crossed to the platform decreased significantly. This indicates that T2DM aggravates memory and cognition disabilities in AD, and may increase the incidence of AD through several mechanisms. These results are consistent with the findings of Zhang et al (16).

In this study, we detected the mTOR activity in hippocampal tissue at the protein and mRNA levels by immunohistochemical analysis and RT-PCR. The mTOR activities in the hippocampal tissue of the T2DM rats increased significantly compared with those of the control group. Thus, T2DM may increase mTOR activity in the brain, possibly by disabling the $\mathrm{PI} 3 \mathrm{~K} / \mathrm{PKB} / \mathrm{mTOR}$ pathway and then increasing IR in the brain. The mTOR levels in the hippocampal tissues of the $\mathrm{T} 2 \mathrm{DM}+\mathrm{AD}$ rats increased significantly compared with those in the T2DM rats, which demonstrates that T2DM triggers excessive mTOR activation in brain tissue, which may aggravate IR in the brain and induce AD.

Furthermore, in this study, we determined the quantity of excessively phosphorylated tau protein by immunohistochemical analysis and the total quantity of tau protein in the hippocampal tissue by RT-PCR. The levels of excessively phosphorylated tau protein and total tau protein significantly increased in the hippocampal tissues of the T2DM+AD rats compared with those in the control, AD and T2DM groups. Thus, T2DM may aggravate the degree of excessive tau protein phosphorylation by activating mTOR activity in the hippocampal tissue and then critically elevate AD. When compared with the control group, tau protein hyperphosphorylation in the hippocampal tissues of the T2DM group rats was not evident. The reason may be that the time of T2DM development is too short or the mTOR activity is not high enough to trigger excessive tau protein phosphorylation. The exact reasons need to be established through further study.

Therefore, the results of the current study indicate that the mechanism by which T2DM increases the risk of AD involves mTOR, a key regulatory protein in the insulin signaling pathway. T2DM causes excessive activation of mTOR in the hippocampal tissue, which may occur through insulin signaling pathway impairment. Although the exces- sive activation of mTOR triggers tau hyperphosphorylation, thereby promoting the occurrence of $\mathrm{AD}$, the relationship between the insulin signaling impairment and the excessive activation of mTOR remains unclear. Further studies on the upstream and downstream factors of mTOR (including PI3K, PKB and S6 kinase 1) may substantially reduce the risk of the co-occurrence of AD and T2DM. Resolving these questions may facilitate the development of new methods for the treatment of AD.

\section{Acknowledgements}

This study was supported by the National Natural Science Foundation of China (No. 81041046).

\section{References}

1. Janson J, Laedtke T, Parisi JE, O'Brien P, Petersen RC and Butler PC: Increased risk of type 2 diabetes in Alzheimer disease. Diabetes 53: 474-481, 2004.

2. Moroz N, Tong M, Longato L, Xu H and de la Monte SM: Limited Alzheimer-type neurodegeneration in experimental obesity and type 2 diabetes mellitus. J Alzheimers Dis 15: 29-44, 2008.

3. Bao CR, Zhou M and Qin DL: Present situation of studies about the relationship between type 2 diabetes and Alzheimer's disease. J Luzhou Medical College 32: 568-569, 2009 (In Chinese).

4. Zhu XY, Niu BF, Dong ZX and Huang HY: Studies of the relationship between type 2 diabetes and Alzheimer's disease. Medical Review 17: 1528-1531, 2011.

5. Chen YJ and Tian JZ: Alzheimer's disease is another form of diabetes. Chin J Gerontology 28: 402-406, 2008 (In Chinese).

6. de la Monte SM and Wands JR: Review of insulin and insulin-like growth factor expression, signaling, and malfunction in the central nervous system: relevance to Alzheimer's disease. J Alzheimer Disease 7: 45-61, 2005.

7. Li Y: The awareness of the relationship between Central insulin resistance and Alzheimer's disease. J Diagn Pract 8: 248-251, 2009.

8. Leibowitz G, Cerasi E and Ketzinel-Gilad M: The role of mTOR in the adaptation and failure of beta-cells in type 2 diabetes. Diabetes 10 (Suppl 4): 157-169, 2008.

9. Glynn EL, Lujan HL, Kramer VJ, Drummond MJ, DiCarlo SE and Rasmussen BB: A chronic increase in physical activity inhibits fed-state mTOR/S6K1 signaling and reduces IRS-1 serine phosphorylation in rat skeletal muscle. Appl Physiol Nutr Metab 33: 93-101, 2008.

10. Vila-Bedmar R, Lorenzo M and Fernández-Veledo S: Adenosine 5'-monophosphate-activated protein kinase-mammalian target of rapamycin cross talk regulates brown adipocyte differ- entiation. Endocrinology 151: 980-992, 2010.

11. Zhang C, Yoon MS and Chen J: Amino acid-sensing mTOR signaling is involved in modulation of lipolysis by chronic insulin treatment in adipocytes. Am J Physiol Endocrinol Metab 296: E862-E868, 2009.

12. Fan H: The research progress of risk-related between diabetes and Alzheimer's disease. J Practical Geriatrics 24: 342-345, 2010.

13. Mi K and Johnson GV: The role of Tau phosphorylation in the pathogenesis of Alzheimer's disease. Curr Alzheimer Res 3: 449-463, 2006.

14. An WL, Cowburn RF, Li L, et al: Up-regulation of phosphorylated/activated p70 S6 kinase and its relationship to neurofibrillary pathology in Alzheimer's disease. Am J Pathol 163: 591-607, 2003.

15. Khurana V, Lu Y, Steinhilb ML, Oldham S, Shulman JM and Feany MB: TOR-mediated cell-cyele activation causes neurodegeneration in a Drosophila tauopathy model. Curr Biol 16: 230-241, 2006.

16. Zhang Y, Zhang Sl, Gao FH, Chen Y and Yang XJ: The research of GSK-3 $\beta$ and tau phosphorylated proteins in hippocampal tissue of type 2 diabetes and Alzheimer's disease rats. Chin J Gerontol 29: 63-66, 2010 (In Chinese). 